\title{
Thyroid hormone abnormalities in euthyroid type 2 diabetes mellitus with chronic kidney disease in Saudi community based hospital
}

\begin{abstract}
Background and objective: The interactions between kidney and thyroid functions are known for years. The present retrospective study was conducted to find out the thyroid hormone abnormalities in patients with chronic kidney disease (CKD) and type 2 diabetes mellitus (T2DM) in Saudi community based hospital.

Design: We analyzed retrospectively 872 participants with T2DM whom are between the age 20 to 96 years. All patients were from the population of the Primary health centre at King Fahad Armed Forces Hospital, Jeddah, Saudi Arabia. All data were collected on the basis of a review of electronic medical data. Participants were defined as having T2DM according to self-report, clinical reports, use of antidiabetic agents and HbAlc $(\geq 6.5)$. HbA1c was expressed as percentage. High performance liquid chromatography was used. Serums FT4 were estimated by radioimmunoassay and Serum TSH was estimated by Immunoradiometric assey. All patients in the present study fulfilled the revised National Kidney Foundation criteria for the diagnosis of CKD. The total numbers of cohort were separated on basis of age values into four groups: $<40$ years, $40-49$ years, 50-59 years and $\geq 60$ years.

Results: 872 subjects with T2DM were included. There were 273 (31.3\%) male and 557 $(68.7 \%)$ were female with mean age $54.5 \pm 13.0$. The mean HbA1c, TSH and FT4 value were $8.1 \pm 2.2,2.0 \pm 1.1 \mathrm{mIU} / 1$ and $15.8 \pm 2.8 \mathrm{pmol} / 1$ respectively. Among cases of T2DM and CKD, there were 239 (61.9\%) female and 147 (38.1\%) were male with female to male ration 1.6:1.0, $<<0.0001$. Patients with $C K D$ were significantly older than patients without CKD, $57.3 \pm 2.9$ vs. $52.3 \pm 12.6$ respectively, $<<0.0001$. Patients with CKD were significantly have higher $\mathrm{HbA} 1 \mathrm{c}$ than patients without $\mathrm{CKD}, 8.7 \pm 2.3$ vs. $7.7 \pm 2.1$ respectively, $\mathrm{p}<0.0001$. Patients with CKD were significantly have higher TSH than patients without CKD, $2.1 \pm 1.0$ vs. $1.9 \pm 1.1$ respectively, $\mathrm{p}=0.001$. Patients with CKD were significantly have lower FT4 than patients without $C K D, 15.5 \pm 2.8$ vs. $16.1 \pm 2.9$ respectively, $p=0.03$. Patients with $C K D$ have higher TSH than patients without CKD across all age groups. Patients with CKD have lower FT4 than patients without CKD across all age groups except patients older than 60years.

Conclusion: We conclude that despite the limitations of this hospital-based retrospective study, high TSH and low FT4 levels are highly prevalent in cohort of Saudis with CKD and T2DM. The majority of our patients in our finding were predominantly females. These two observations remain to be validated by population-based studies. In the absence of registry data, larger cooperative studies involving diverse population samples from multiple centers could help to provide further information on the true thyroid hormone abnormalities.
\end{abstract}

Volume 6 Issue 2 - 2019

\author{
Khalid S Aljabri,', Ibrahim MAlnasser,'2 Samia \\ A Bokhari,' Muneera A Alshareef ,' Patan \\ M Khan,' Abdulla M Mallosho,' Hesham M \\ Abu Elsaoud,' Mohammad M Jalal,' Rania F \\ Safwat,' Rehab El Boraie,' Nawaf K Aljabri, ${ }^{3}$ \\ Bandari K Aljabri, ${ }^{4}$ Arwa Y Alsuraihi MS, ${ }^{4}$ \\ Amjad I Hawsawi ${ }^{4}$ \\ 'Department of Endocrinology, King Fahad Armed Forces \\ Hospital, Saudi Arabia \\ ${ }^{2}$ Department of Radiology, King Fahad Armed Forces Hospital, \\ Saudi Arabia \\ ${ }^{3}$ Department of Laboratory, Northern Armed Forces Hospital, \\ Saudi Arabia \\ ${ }^{4}$ College of medicine, Um Al Qura University, Saudi Arabia
}

Correspondence: Khalid S Aljabri, Department of Endocrinology, King Fahad Armed Forces Hospital, Saudi Arabia, Tel +9662323333, Email khalidsaljabri@yahoo.com

Received: February 03, 2019 | Published: April 02, 2019

Keywords: Chronic kidney disease, Thyroid hormones, Type 2 Diabetes

\section{Introduction}

Saudi Arabia is the seventh of the top ten countries in terms of the prevalence of type 2 diabetes mellitus (T2DM) among Saudi adults older than 20 years. ${ }^{1}$ According to the 2010 Global Burden of Disease study, chronic kidney disease (CKD) was ranked $27^{\text {th }}$ in the list of causes of total number of deaths worldwide in 1990 , but rose to 18 th in $2010 .^{2}$

The interactions between kidney and thyroid functions are known for years. ${ }^{3-6}$ Thyroid hormones (TH) are necessary for growth and development of the kidney and for the maintenance of water and electrolyte homeostasis. On the other hand, kidney is involved in the metabolism and elimination of TH. Moreover, the decline of kidney function is accompanied by changes in the synthesis, secretion, metabolism, and elimination of TH. Thyroid dysfunction acquires special characteristics in those patients with advanced kidney disease. ${ }^{7}$

The two main hormones produced by the thyroid are triiodothyronine (T3) and thyroxine (T4). These hormones can also have significant impact on kidney disease so it is important to consider the physiological association of thyroid dysfunction in relation to CKD. Thyroid stimulating hormone (TSH) which is biosynthesized in pituitary is responsible for every single biochemical process within thyroid gland including T4 and T3 production. $^{7}$ It seems eventually kidney is involved in key pathways of the T4 and T3 metabolism and any condition with eventual renal dysfunction may lead to thyroid disorder and this abnormality can be manifested through TH production and metabolism. ${ }^{8,9}$ However, most patients with CKD are euthyroid, with normal TSH and free T4 levels. 
Gilles et al, made the interesting observation that patients with CKD had higher TSH levels, which can be explained by the possible loss of thyroid hormones in the urine..$^{10}$ In addition, thyroid hormone abnormalities have been reported among euthyroid patients with end stage renal failure (ESRD), including reduced total and free triiodothyronine and thyroxine levels. ${ }^{11}$ Reasons for these latter findings are unclear, but it has been postulated to be due, at least in part, to an adaptive response to chronic nonthyroidal illness, unresolved uremia, and protein malnutrition. ${ }^{12}$ Thus, the present retrospective study was conducted to find out the thyroid hormone abnormalities in patients with CKD and T2DM in Saudi community based hospital.

\section{Methods}

We analyzed retrospectively 872 participants whom are between the age 20 to 96years. All patients were from the population of the Primary health centre at King Fahad Armed Forces Hospital, Jeddah, Saudi Arabia. All data were collected on the basis of a review of electronic medical data. Participants were defined as having T2DM according to self-report, clinical reports, use of antidiabetic agents and HbAlc $(\geq 6.5) .{ }^{13} \mathrm{HbA} 1 \mathrm{c}$ was expressed as percentage. High performance liquid chromatography was used. Serums FT4 were estimated by radioimmunoassay and Serum TSH was estimated by Immunoradiometric assey. All patients in the present study fulfilled the revised National Kidney Foundation criteria for the diagnosis of CKD. ${ }^{14,15}$ The total number of cohort were separated on basis of age values into four groups: <40years, 40-49years, 50-59years and $\geq 60$ years.

\section{Statistical analysis}

Continuous variables were described using means and standard deviations. Univariate analysis of baseline demography both between groups, were accomplished using unpaired t-test and Chi square test were used for categorical data comparison. Pearson correlation was used for correlation. $\mathrm{P}$ value $<0.05$ indicates significance. The statistical analysis was conducted with SPSS version 22.0 for Windows.

\section{Results}

872 subjects with T2DM were included. There were $273(31.3$ $\%)$ male and 557 (68.7\%) were female with mean age 54.5 \pm 13.0 , Table 1. The mean HbAlc, TSH and FT4 value were $8.1 \pm 2.2$, $2.0 \pm 1.1 \mathrm{mIU} / 1$ and $15.8 \pm 2.8 \mathrm{pmol} / 1$ respectively. Among cases of T2DM and CKD, there were 239 (61.9\%) female and $147(38.1 \%)$ were male with female to male ration 1.6:1.0, $\mathrm{p}<0.0001$. Patients with CKD were significantly older than patients without CKD, $57.3 \pm 12.9$ vs. $52.3 \pm 12.6$ respectively, $\mathrm{p}<0.0001$. Patients with $\mathrm{CKD}$ were significantly have higher $\mathrm{HbAlc}$ than patients without $\mathrm{CKD}$, $8.7 \pm 2.3$ vs. $7.7 \pm 2.1$ respectively, $p<0.0001$. Patients with $C K D$ were significantly have higher TSH than patients without CKD, $2.1 \pm 1.0$ vs. $1.9 \pm 1.1$ respectively, $p=0.001$. Patients with $C K D$ were significantly have lower FT4 than patients without CKD, $15.5 \pm 2.8$ vs. $16.1 \pm 2.9$ respectively, $\mathrm{p}=0.03$. Patients with $\mathrm{CKD}$ have higher TSH than patients without CKD across all age groups, (Figure 1). Patients with CKD have lower FT4 than patients without CKD across all age groups except patients older than 60 years, (Figure 2).

Table I Characteristic of patients with type 2 diabetes with and without chronic kidney disease [mean \pm standard deviation or number (\%)]

\begin{tabular}{|c|c|c|c|c|c|}
\hline \multirow{2}{*}{ Parameters } & & \multirow{2}{*}{ Total } & \multicolumn{2}{|c|}{ Chronic kidney disease } & \multirow{2}{*}{$P$ value } \\
\hline & & & Present & Absent & \\
\hline Numbers & & 872 & $386(44.3)$ & $486(55.7)$ & ( \\
\hline Age (years) & & $54.5 \pm 13.0$ & $57.3 \pm 12.9$ & $52.3 \pm 12.6$ & 0.0001 \\
\hline \multirow{2}{*}{ Gender } & Male & $273(3 \mid .3)$ & $147(38.1)$ & $126(25.9)$ & \multirow{2}{*}{$<0.0001$} \\
\hline & Female & $557(68.7)$ & $239(61.9)$ & $360(74.1)$ & \\
\hline $\mathrm{HbAlc}$ & & $8.1 \pm 2.2$ & $8.7 \pm 2.3$ & $7.7 \pm 2.1$ & $<0.0001$ \\
\hline TSH (mlU/l) & & $2.0 \pm 1.1$ & $2.1 \pm 1.0$ & $1.9 \pm 1.1$ & 0.001 \\
\hline FT4 (pmol/l) & & $15.8 \pm 2.8$ & $15.5 \pm 2.8$ & $16.1 \pm 2.9$ & 0.03 \\
\hline Serum creatinine $(\mu \mathrm{mol} / \mathrm{L})$ & & $71.0 \pm 28.0$ & $79.6 \pm 36.7$ & $64.2 \pm 15.3$ & $<0.0001$ \\
\hline
\end{tabular}

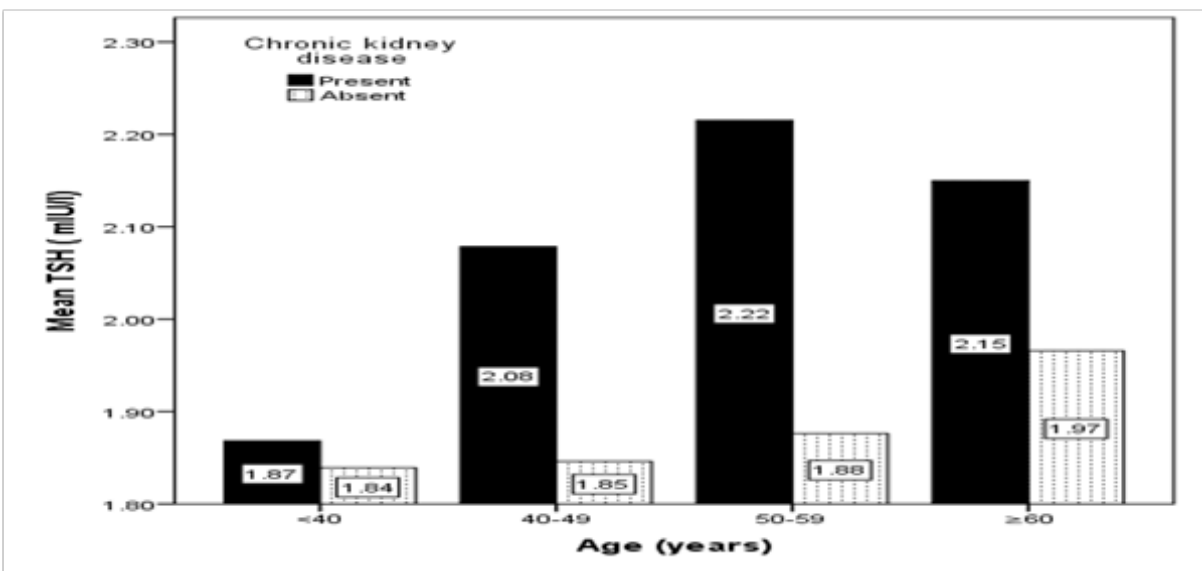

Figure I Age category groups and the mean TSH in patients with type 2 diabetes with and without chronic kidney disease. 


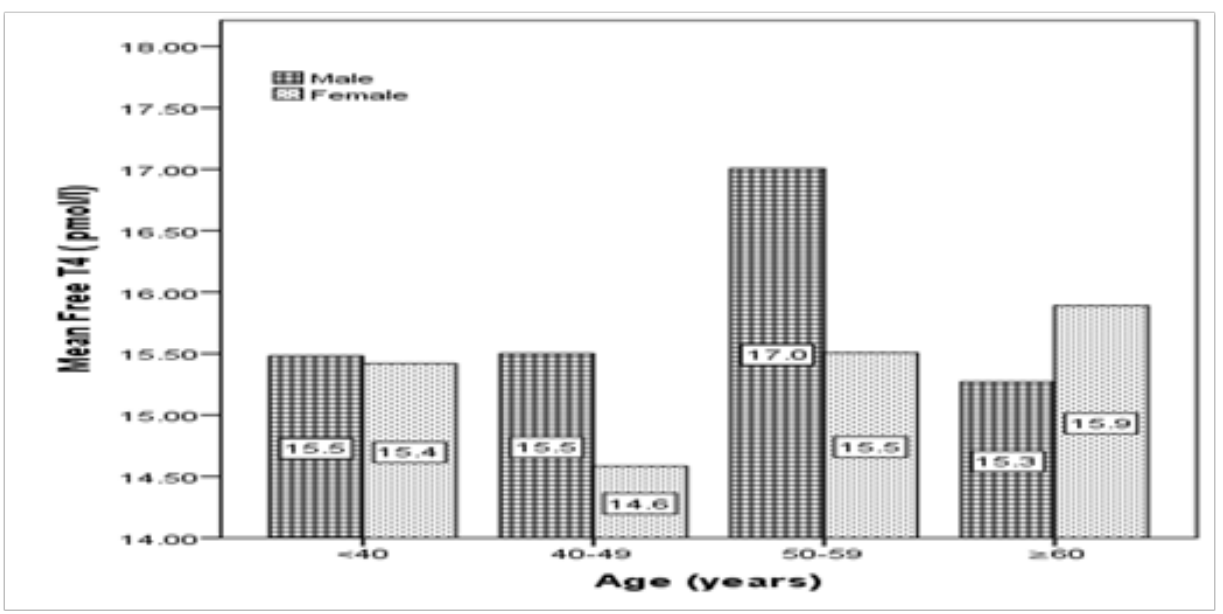

Figure 2 Age category groups and the mean Free T4 in patients with type 2 diabetes with chronic kidney disease.

\section{Discussion}

Our study demonstrated that abnormalities in thyroid function occur in patients with T2DM complicated by CKD. Specifically, TSH levels were higher and FT4 levels were lower in patients with diabetic renal diseases. CKD implies progressive, long-standing and irreversible impairment in renal function that results in ESRD. ${ }^{16}$ The interplays between thyroid and kidney have been recognized in many disease states. Similarly, kidney is also involved in the regulation of thyroid hormones metabolism. ${ }^{17}$ Thyroid dysfunction can influence kidney through the immune-mediated pathway and thyroid hormones. ${ }^{18}$ The thyroid disorder and coincident nephropathy have been reported mainly in the patients presented with CKD. ${ }^{19,20}$ In 2009, Iglesias P and Diez JJ published a review article entitled thyroid dysfunction and kidney disease in a European Journal of Endocrinology in which they report that serum TSH concentrations are usually normal or elevated in CKD. Free and total T4 concentrations are usually normal or low in patients with CKD. ${ }^{21}$ Disorders of thyroid function have also been linked to development of immune mediated glomerular injury and alterations in thyroid hormones and thyroid hormone testing occur in patients with kidney disease.

Higher TSH levels are seen with increasing age in our study. Surks et al showed in patients without thyroid disease, $10.6 \%$ of 20 - to 29 -yrolds had TSH greater than $2.5 \mathrm{mIU} / \mathrm{liter}$, increasing to $40 \%$ in the $80+$ group, $14.5 \%$ of whom had TSH greater than $4.5 \mathrm{mIU} / \mathrm{liter}^{22}$ The TSH level is often elevated in CKD in response to TSH from pituitary as a result of uremic effect. ${ }^{23}$ In disagreement to our study, patients with HbAlc was no significantly negatively correlated TSH levels, $r=0.05$, $\mathrm{p}=0.2 .^{24} \mathrm{TSH}$ also loses its circadian rhythm along with compromised bioactivity due to poor glycosylation. The Wolffe Chaikoff effect has been cited as a causative phenomenon behind the rise of this disorder in diabetic kidney disease patients. ${ }^{25}$

In agreement to our study, we found that patients with CKD were significantly have lower FT4 than patients without CKD, NA and MI, $15.5 \pm 2.8$ vs. $16.1 \pm 2.9$ respectively, $\mathrm{p}=0.03 .{ }^{10}$

CKD affects thyroid function in several ways that include low circulating thyroid hormone concentration, alteration in peripheral hormone metabolism, and disturbance in binding them to carrier proteins, possible reduction in tissue thyroid hormone content and increased iodine store in thyroid gland resulting in the reduction of serum T4. ${ }^{12}$ Normal or low levels of T4 may be due to the monodeiodinase action occurring in the inner benzene ring instead of outer ring of T4, resulting in the formation of reverse T3. ${ }^{12}$
We aimed to identify the levels of thyroid hormones in euthyroid T2DM patients complicated with CKD in primary health care setting. Furthermore, due to the retrospective nature of this study, the observed population reflects a selected yet comprehensive group of patients rather than the general population. Our study could be limited by the question of clustering of cases within the study region and the effect that might have on our estimates, in addition, the current study population may appear limited in size and therefore may underestimate the true thyroid hormone abnormailities in patients with T2DM complicated with CKD. In addition, the study shares the limitations of all retrospective studies.

We conclude that despite the limitations of this hospital-based retrospective study, high TSH and low FT4 levels are highly prevalent in cohort of Saudis with CKD and T2DM. The majority of our patients in our finding were predominantly females. These two observations remain to be validated by population-based studies. In the absence of registry data, larger cooperative studies involving diverse population samples from multiple centers could help to provide further information on the true thyroid hormone abnormalities.

\section{Acknowledgment}

The author would like to thank all colleagues from the Department of primary care for helping in data collection.

\section{Conflicts of interests}

The authors declare no conflict of interests.

\section{References}

1. IDF Diabetes Atlas 5th edition 2012 update. International Diabetes Federation. 2011.

2. Feinstein EI, Kaptein EM, Nicoloff JT, et al. Thyroid function in patients with nephrotic syndrome and normal renal function. American Journal of Nephrology. 1982;2:70-76.

3. Kaptein EM, Quion VH, Massry SG. Hemodynamic effects of thyroid hormone. Contributions to Nephrology. 1984;41:151-159.

4. Kaptein EM. Thyroid function in renal failure. Contributions to Nephrology. 1986;50:64-72.

5. Kaptein EM, Feinstein EI, Massry SG. Thyroid hormone metabolism in renal diseases. Contributions to Nephrology. 1982;33:122-135.

6. Kaptein EM, Quion VH, Chooljian CJ, et al. The thyroid in endstage renal disease. Medicine 1988;67:187-197. 
7. Kaptein EM, Hoopes MT, Parise M, et al. rT3 metabolism in patients with nephrotic syndrome and normal GFR compared with normal subjects. Am J Physiol. 1991;260:641-650.

8. Katz AI, Emmanouel DS, Lindheimer MD. Thyroid hormone and the kidney. Nephron. 1975;15:223-249.

9. Gilles R, den Heijer M, Ross AH, et al. Thyroid function in patients with proteinuria. Neth J Med. 2008;66:483-485.

10. Hegedus L, Andersen JR, Poulsen LR, et al. Thyroid gland volume and serum concentrations of thyroid hormones in chronic renal failure. Nephron. 1985;40:171-174.

11. Lim VS. Thyroid function in patients with chronic renal failure. Am J Kidney Dis. 2001;38:80-84.

12. American Diabetes Association. Classification and Diagnosis of Diabetes: Standards of Medical Care in Diabetes-2019. Diabetes Care. 2019;42(1):13-28.

13. National Kidney Foundation. K/DOQI Clinical Practice Guidelines for Chronic Kidney Disease. Part 5: Evaluation of laboratory measurements for clinical assessment of kidney disease. Am J Kidney Dis. 2002;39:76-92.

14. Isakova T, Nickolas TL, Denburg M, et al. KDOQI US Commentary on the 2017 KDIGO Clinical Practice Guideline Update for the Diagnosis, Evaluation, Prevention, and Treatment of Chronic Kidney DiseaseMineral and Bone Disorder (CKD-MBD). American Journal of Kidney Diseases. 2017;70(6)737-751.

15. Parmar MS. Chronic renal disease. BMJ. 2002;325(7355):85-90.
16. Ramirez G, Neill W, Jubiz W, et al. Thyroid dysfunction in uremia: evidence for thyroid and hypophyseal abnormalities. Ann Int Med. 1996;84:672-676.

17. Basu G, Mohapatra A. Interactions between thyroid disorders and kidney disease. Indian J Endocrinol Metab. 2014;16:204-214.

18. Kocak G, Huddam B, Azak A, et al. Coexistent findings of renal glomerular disease with Hashimoto's thyroiditis. Clin Endocrinol (Oxf). 2012;76:759-762.

19. Hao Y, Wu X, Huang X, et al. Proteinuria Associated with Thyroid Disorders-Cases Report and Literature Review. Med Case Rep. 2017;3(4):1-4.

20. Iglesias P, Diez JJ. Thyroid dysfunction and kidney disease. Eur J Endocrinol. 2009;160(4):503-315.

21. Surks MI, Hollowell JG. Age-specific distribution of serum thyrotropin and antithyroid antibodies in the US population: implications for the prevalence of subclinical hypothyroidism. J Clin Endocrinol Metab. 2007;92:4575-4582.

22. Ramirez G, Neill W, Jubiz W, et al. Thyroid dysfunction in uremia: evidence for thyroid and hypophyseal abnormalities. Ann Intern Med. 1976;84:672-676.

23. Chandra A. Prevalence of hypothyroidism in patients with chronic kidney disease: a cross-sectional study from North India. Kidney Res Clin Pract. 2016;35(3):165-168.

24. Bando Y, Ushiogi Y, Okafuji K, et al. Nonautoimmune primary hypothyroidism in diabetic and non-diabetic chronic renal dysfunction. Exp Clin Endocrinol Diabetes. 2002;110:408-415. 